\title{
Search for correlations between BATSE Gamma-Ray Bursts and Supernovae $\left(^{*}\right)$
}

\author{
J. Polchr $\left({ }^{1}\right)$, M. Topinka $\left({ }^{2}\right)\left({ }^{* *}\right)$, D. NeČas $\left({ }^{3}\right)$, F. Hroch $\left({ }^{1}\right)$, V. Hudcová $\left({ }^{2}\right)$, \\ R. Hudec $\left({ }^{2}\right)$, G. Pizzichini $\left({ }^{4}\right)$, N. Masettit $\left({ }^{4}\right)$ and E. Palazzi $\left({ }^{4}\right)$ \\ $\left({ }^{1}\right)$ Masaryk University Brno, Faculty of Science, Czech Republic \\ $\left({ }^{2}\right)$ Astronomical Institute of the Academy of Sciences of Czech Republic, Ondřejov, \\ Czech Republic \\ $\left(^{3}\right)$ Brno University of Technology, Faculty of Civil Engineering, Czech Republic \\ $\left.{ }^{4}\right)$ C.N.R. Instituto di Astrofisica Spaziale e Fisica Cosmica - I.A.S.F./CNR, Bologna, Italy
}

(ricevuto il 23 Maggio 2005; pubblicato online il 2 Novembre 2005)

\begin{abstract}
Summary. - We report on complex statistical research of space-time correlated supernovae and CGRO-BATSE gamma-ray bursts. We show that there exists a significantly higher abundance of core-collapse supernovae among the correlated supernovae, but the subset of all correlated objects does not seem to be physically different from the whole set.
\end{abstract}

PACS 98.70.Rz - $\gamma$-ray sources; $\gamma$-ray bursts.

PACS 97.60.Bw - Supernovae.

PACS 01.30.Cc - Conference proceedings.

\section{1. - Introduction}

The origin and source of gamma-ray bursts (GRBs) still remain a puzzle. This tremendous energy could be released on a short time-scale during a collapse of a massive star in a supernova-like explosion. There are several pieces of observational evidence supporting the connection between GRBs and supernovae ( $\mathrm{SNe}$ ): some GRBs reveal an underlying $\mathrm{SN}$ in an optical afterglow lightcurve (e.g. GRB980326), sometimes supported by spectral and colour signatures (e.g. GRB030329 and SN2003dh), and there are a few objects with space-time coincidence (e.g. GRB980425 and SN1998bw).

We have used the current CGRO-BATSE GRB catalogue (up to date version to April 2004) [2] and combined data from Asiago Padova [4], Harvard [3] and Sternberg [1] catalogues of SNe.

(*) Paper presented at the "4th Workshop on Gamma-Ray Burst in the Afterglow Era", Rome, October 18-22, 2004.

$\left.{ }^{* *}\right)$ Please for any details, comments or suggestions to this proceeding contact J. Polcar (polcar@physics.muni.cz) or M. Topinka (toast@asu.cas.cz)

(C) Società Italiana di Fisica 
TABLE I. - Clasification of types of supernovae.

\begin{tabular}{|c|c|}
\hline unknown & 'NULL', , \\
\hline core & $\begin{array}{l}\text { ib', 'ib, 'ic', 'ic, 'ic', 'ib/c', 'ic-p', } \\
\text { 'ii', 'ii , 'iib', 'iib , 'iic', 'iic, 'ii-n', } \\
\text { ii-n, 'ii-l', 'ii-p', 'ii-p, 'iipec' }\end{array}$ \\
\hline dwarf & 'ia', ,'ia, 'ia-p', 'ia-p, 'i', 'i, 'i-p', \\
\hline type1 & $\begin{array}{l}\text { 'i', 'i, 'i-1', 'ia', 'ia, 'ia-p', 'ia-p, } \\
\text { ib', 'ib, 'ic', 'ic, 'ib/c', 'ic-p' }\end{array}$ \\
\hline type2 & $\begin{array}{l}\text { 'ii', 'ii-1', 'ii, 'iib', 'iib, 'iic', 'iic, } \\
\text { 'ii-1', 'iipec' }\end{array}$ \\
\hline plateau & 'ia-p', 'ia-p, 'ic-p', 'ii-p', 'ii-p, ' $i-p$ ' \\
\hline linear & 'i-1', 'ii-1' \\
\hline narrow & 'ii-n', 'ii-n \\
\hline bizzare & 'iipec' \\
\hline uncertain & ' $\left[\left[^{\sim} \backslash \backslash\right.\right.$, \\
\hline
\end{tabular}

\section{2. - Pseudotypes}

There is a wide range of the SN types existing in the input data. For easier manipulation we have divided all the SNe into several types, called pseudotypes. Note that the division is not disjunct. See table I. For the purposes of our study it is useful to simplify this division into three pseudotypes only: the dwarf, the core and the unknown SNe.

\section{3. - Space and time correlation}

The situation is easy in the space domain, A GRB and a SN make a pair if the location of the SN falls into the GRB errorbox which yield $3 \sigma$ error. In the time domain it is not a priori theoretically clear what is the time delay between the SN and an possible gamma emission. For our purposes we postulated the time of the optical emission to be the time of the maximum $T_{\max }$ of the optical lightcurve of the SN. Unfortunately only a fraction of the SNe provides an information about the date of $T_{\max }$. We solved the problem statistically and we assumed the time delay between $T_{\max }$ and the time of the discovery $T_{\text {disc }}$ to be the median $M_{\max }=-4$ days. We get it from the distribution (fig. 1) of all known time delays. Thus for the SNe which have the time of the maximum missing

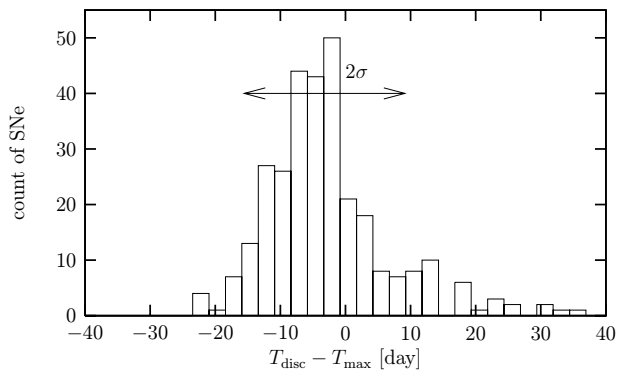

Fig. 1. - Distribution of $T_{\text {dist }}-T_{\max }$ delay. 

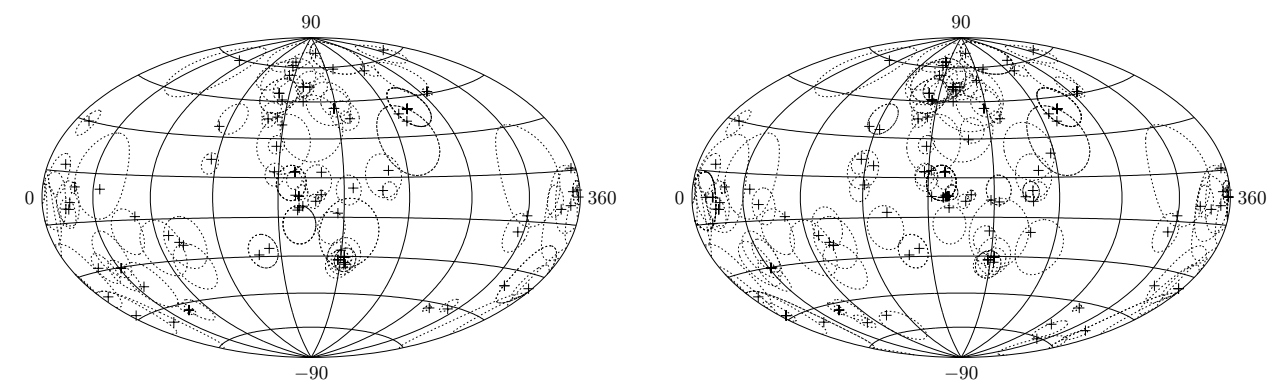

Fig. 2. - Matched pairs for $\mathbb{A}$ (left) and $\mathbb{B}$ case.

we computed it as

$$
T_{\max }=T_{\text {disc }}+M_{\max } .
$$

The time delay $T_{\delta}$ between the possible prompt gamma emission $T_{\gamma}$ of the SN and its optical emission $T_{\max }$ may depend on the type of the SN. We respect it by the division into the pseudotypes. We represent the time delay by the shift in time $T_{\delta}$ which is the mean $\mu_{\gamma}$ of the Gaussian distribution function and by the half-width of the time window $T_{\text {error }}$ which is $3 \sigma_{\gamma}$ error in the term of the Gaussian. Generally it could be written as

$$
T_{\gamma}=\left(T_{\max }+\mu_{\gamma}\right) \pm 3 \sigma_{\gamma} .
$$

We tried to make the best possible estimate. The typical time delay $T_{\delta}$ can be estimated for the dwarf SN as a relatively narrow time interval $+20 \pm 7$ days [5]. For the corecollapse $\mathrm{SNe}$ we choose the typical values of the time delay to be $0 \pm 30$ days.

For each pseudotype we assume the Gaussian distribution of the time delays $T_{\gamma}-T_{\max }$. We calculated the weighted average of the particular densities of the probability for every configuration with respect to the level of uncertainty we had. For the match we used the best Gaussian fit of the density of the probability. The matching process itself reflects the effect of the selection of the SNe into the pseudotypes, it results into the different size of the time error used for each pseudotype. The larger time-errorbox of the particular pseudotype we have the greater number of matched pairs of this kind we expect. To compare this effect it is useful to do the matching separately for two different sets of settings: $\mathbb{A}$ for the one which is dependent on the pseudotype of the SN and $\mathbb{B}$ for which is not.

\section{4. - Number of matched pairs}

We rotated the SN catalogue around arbitrary axes (295 different rotation). In both cases $(\mathbb{A}$ and $\mathbb{B})$ we found that the number of matched pairs is less than the average number of matched pairs as they were derived from the rotations but in both cases $\mathbb{A}$ and $\mathbb{B}$ within $1 \sigma$ error.

\section{5. - Relative abundance of pseudotypes}

We studied the spectrum of SN pseudotypes in the set of possibly correlated SNe with respect to all SNe. We test if the relative abundance of the core SNe differs from the 

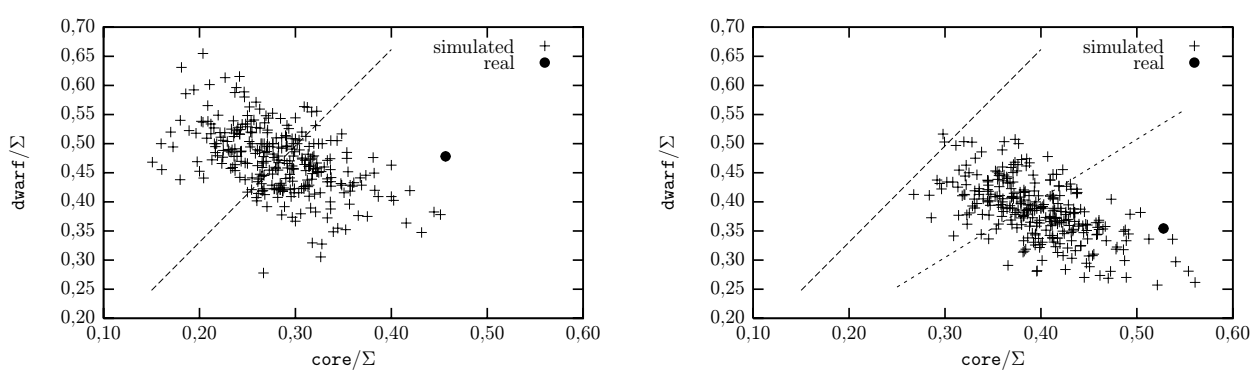

Fig. 3. - Relative abundance of pseudotypes for $\mathbb{A}$ (left) and $\mathbb{B}$ case.

whole SN catalogue. To exclude the various possible selection effect and to prove the higher number of the core SNe among the coinciding pairs we compare the results of the real match with artificial random SN and GRB distribution we can generate using the rotation method. In the case of artificially build catalogues we see a significant difference between the $\mathbb{A}$ and $\mathbb{B}$ sets of parameters (fig. 3). It is due to the different size of the time window. However, the relative abundance of the core $\mathrm{SNe}$ in the real match still remains different from the distribution of the relative abundances in the random cases. The distribution of the abundances of the core SNe in the artificial data set is Gaussian up to $97.8 \%$ in the meaning of Kolmogorov-Smirnov test and the relative abundance of the core $\mathrm{SNe}$ in the real match is out of the $3 \sigma$ interval.

\section{6. - Conclusion}

We have carried out a complex analysis of space-time correlated SNe and CGROBATSE GRBs. We have compared count of matched possible correlated pairs SN-GRB with a random one and have estimated the detectable fraction of physically and random correlated pairs. There exists a significantly higher abundance of core-collapse supernovae among the correlated supernovae, but the subset of all correlated objects does not seem to be physically different from the whole set. A good test of the relevance of this result would be to derive a pseudo-redshift for the possibly correlated BATSE bursts using Amati's relation [8] and compare them with the redshifts of the matched SNe.

We acknowledge the support by the Grant Agency of the Academy of Sciences of the Czech Republic, grant A3003206 and 205/03/H144.

\section{REFERENCES}

[1] http://www.sai.msu.su/sn/

[2] http://cossc.gsfc.nasa.gov/batse/index.html

[3] http://cfa-www.harvard.edu/iau/lists/Supernovae.html

[4] http://merlino.pd.astro.it/ supern/snean.txt

[5] PetscheK A. G., Supernovae Springer Verlag New York 1996.

[6] Vietri M. and Stella L., ApJ, 507 (1998) 45.

[7] MacFayeden A. and Woosley I., ApJ, 524 (1999) 262.

[8] Amati L. et al., A\&A, 390 (2002) 81. 